$$
S_{n}{ }^{(k)}, \quad n S_{n}{ }^{(k-1)}, \quad n^{2} S_{n}^{(k-2)}, \quad \cdots, \quad n^{k-1} S_{n}{ }^{(1)}
$$

is in absolute value less than $B$ for every value of $n$. Suppose further that the series

$$
\sum_{i=1}^{\infty}\left|\Delta^{k} c_{i}\right|
$$

converges uniformly in $D$ and

Then the series

$$
\lim _{n=\infty} \Delta^{k-1} c_{n}=0 \text {. }
$$

$$
a_{1} c_{1}+a_{2} c_{2}+a_{3} c_{3}+\cdots
$$

converges uniformly in $D$.

For the case $k=1$ both of these theorems are already known. This special case of Theorem III appears to have been first employed by Nielsen*; but Nielsen's statement of it is not entirely accurate, as Landau $\dagger$ has pointed out. The corresponding special case of Theorem IV is due to Cahen.

INDIANA UNIVERSITY, July, 1913.

\title{
A TRANSLATION PRINCIPLE CONNECTING THE INVARIANT THEORY OF LINE CONGRUENCES WITH THAT OF PLANE $n$-LINES.
}

\author{
BY PROFESSOR O. E. GLENN.
}

(Read before the American Mathematical Society, September 9, 1913.)

A weLL known translation principle of Clebsch§ enables us to construct a ternary invariant by a simple algebraical operation upon a binary invariant. In brief, if the line $u_{x}=0$ intersects the curve

$$
f=a_{x}{ }^{n}=b_{x}{ }^{n}=\cdots=0
$$

in the $n$ points given by the binary form

* Annali di Matematica (3), 15 (1908), pp. 275-282.

† Sitzungsber. d. K. Bayer. Akad. d. Wiss., Phys.-Math. Classe, 1909.

$\ddagger$ Annales scientifiques de l'Ecole Normale Supérieure (3), 11 (1894), p. 79.

$\S$ Clebsch, Vorlesungen über Geometrie, vol. 1, p. 276; and Journal für Math., vol. 59 (1861). 
and if

$$
g=a_{\xi}{ }^{\prime n}=b_{\xi}{ }^{\prime n}=\cdots=0,
$$

$$
I=\Sigma C\left(a^{\prime} b^{\prime}\right)\left(a^{\prime} c^{\prime}\right) \cdots
$$

is any invariant of $g$, then

$$
I(u)=\Sigma C(a b u)(a c u) \cdots
$$

is a contravariant of $f$; and it represents the envelope of $u_{x}=0$ when the latter cuts $f=0$ in a range having the projective property $I=0$.

Let us, for the moment, call $f=0, g=0$ the higher and lower locus, respectively, and $u_{x}=0$ the joining locus. Then a translation principle is an algebraical process depending upon the joining locus, which converts an invariant of the lower locus into an invariant of the higher locus.

In this note we establish a translation principle in which the higher locus is a line congruence in three-space, the lower locus a plane $n$-line, and the joining locus is a plane.

\section{$\S 1$. The Congruence $C(n ; 1)$.}

The line determined by two planes

$$
u_{x} \equiv x_{1}-p_{1} x_{3}-q_{1} x_{4}=0, \quad v_{x} \equiv x_{2}-p_{2} x_{3}-q_{2} x_{4}=0
$$

was designated by Plücker* as his element of three-space. As coordinates of one of the $\infty^{4}$ elements we have the nonhomogeneous system

$$
\left(p_{1}, q_{1}, p_{2}, q_{2}, r\right) \quad[r=(p q)] .
$$

A non-homogeneous form in these variables represents $\infty^{3}$ elements forming a line complex. Consider two such forms of orders $n$ and unity respectively,

$$
\begin{gathered}
f_{n}(p, q, r)=0, \\
f_{1}(p, q, r) \equiv \sum_{i=1}^{2}\left(\alpha_{i} p_{i}+\beta_{i} q_{i}\right)+\gamma_{1} r+\gamma_{2}=0 ;
\end{gathered}
$$

the $\infty^{2}$ elements common to these two complexes form a line congruence which we may represent by $C(n ; 1)$.

The intersection of an arbitrary plane $w_{x}=0$ and the congruence $C(n ; 1)$ is an n-line.

* Plücker, System der Geometrie des Raumes u. s. w. (1846). 
An analytical proof is furnished as follows: Impose the condition that $w_{x}=0$ pass through the element $\left(u_{x}, v_{x}\right)$. This is

or

$$
\left\|\begin{array}{llll}
w & w_{2} & w_{3} & w_{4} \\
-1 & 0 & p_{1} & q_{1} \\
0 & -1 & p_{2} & q_{2}
\end{array}\right\|=0,
$$

(3) $\quad p_{1} w_{1}+p_{2} w_{2}+w_{3}=0, \quad q_{1} w_{1}+q_{2} w_{2}+w_{4}=0$.

From (3) we get

$$
r \equiv(p q)=\frac{1}{w_{1}}\left(w_{4} p_{2}-w_{3} q_{2}\right),
$$

and the substitution of this in $f_{1}$ eliminates $r$ and reduces $f_{1}$ to a linear non-homogeneous form in $p_{i}, q_{i}(i=1,2)$, involving the parameters $w$.

We now solve $f_{1}(p, q)$ with (3), thus expressing $p_{2}, q_{1}, q_{2}$ each as a linear expression in $p_{1}$, say

$$
\begin{aligned}
& p_{2}=g(w) p_{1}+h(w), \\
& q_{i}=g_{i}(w) p_{1}+h_{i}(w) \quad(i=1,2) .
\end{aligned}
$$

Substitute these in $f_{n}(p, q)$ and we get a non-homogeneous equation or form $F\left(p_{1}\right)$ in $p_{1}$, with coefficients rational in $w_{i}(i=1,2,3,4)$, and of order $n$. The $n \operatorname{roots} p_{1}^{(j)}(j=1$, $2, \cdots, n)$ put in (5) in turn, give us just $n$ sets

$$
\left(p_{i}{ }^{(j)}, q_{i}{ }^{(j)}, r^{(j)}\right) \quad(i=1,2 ; j=1,2, \cdots, n) .
$$

These sets are the coordinates of $n$ lines of intersection as stated.

Now if we project the $n$-line in which $w_{x}=0$ cuts $C(n ; 1)$ upon the $x_{3}=0$ plane, we get the $n$-line whose equation is

$$
f_{3 n} \equiv \prod_{j=1}^{n}\left(p_{2}{ }^{(j)} x_{1}-p_{1}{ }^{(j)} x_{2}+r^{(j)} x_{4}\right)=0 .
$$

In the translation principle to be established $C(n ; 1)$ figures as the higher locus, $f_{3 n}=0$ as the lower locus, and $w_{x}=0$ the joining locus.

\section{§2. Translation Principle.}

It is easy to show by combining (4), (5), (6) that the $n$ lines of $f_{3 n}$ are concurrent. Some of the full invariants of 
this $n$-line therefore vanish identically. Thus when $n=3$ the only full invariant, the condition for a triple point, vanishes identically. Now, however, even when $n=3$ or $n=2$ we always have non-vanishing invariants of the binary form $F\left(p_{1}\right)$, and these, through (6), have interpretations as invariants of the lower locus $f_{3 n}$. For instance, if the discriminant of $F\left(p_{1}\right)$ vanishes, then $f_{3 n}$ has a double line.

Every rational invariant $I$ of $F\left(p_{1}\right)$ is a rational form in $w_{i}(i=1,2,3,4)$. Such a form represents an algebraical surface whose equation is in planar coordinates. This surface, $\varphi(w)=0$, is the envelope of the plane $w_{x}=0$ when the latter moves so as to cut the congruence $C(n ; 1)$ in the $n$-line having the projective property $I=0$.

It is to be noted that $q_{1}, q_{2}, p_{2}$ each satisfy an equation analogous to $F\left(p_{1}\right)=0$. But each equation may be obtained from $F\left(p_{1}\right)=0$ by a homographic transformation of $F\left(p_{1}\right)$, as is readily shown from (1), (3), (4). The determinants of these transformations will be different from zero, save when (1) and (3) are inconsistent. Hence the surfaces obtained from the equations in $q_{i}, p_{2}$ coincide with $\varphi(w)=0$. In other words the translation principle gives a unique result, $\varphi(w)$.

\section{§3. Class of $C(n ; 1)$, etc.}

A conspicuous case of $I$ is the discriminant of $F\left(p_{1}\right)$. Applying the translation principle, we get a surface $\varphi(w)=0$ which is the envelope of $w_{x}=0$ when the latter moves so as to touch $C(n ; 1)$ in a double line. In this case $\varphi(w)=0$ may be called the class equation of the congruence $C(n ; 1)$. The order of $\varphi(w)$ is the class of $C(n ; 1)$.

For illustration we take the special congruence given by

$$
\begin{aligned}
& f_{2}(p, q) \equiv(p q)+p_{1} q_{1}+a p_{2}=0, \\
& f_{1}(p, q) \equiv(p q)+1=0 .
\end{aligned}
$$

By (3), (4) we have

Hence

$$
\begin{gathered}
w_{4} p_{2}-w_{3} q_{2}+w_{1}=0, \\
w_{1} p_{1}+w_{2} p_{2}+w_{3}=0, \\
w_{1} q_{1}+w_{2} q_{2}+w_{4}=0 .
\end{gathered}
$$

(8) $\quad F\left(p_{1}\right) \equiv w_{2} w_{4} p_{1}^{2}-\left(w_{2}^{2}+a w_{1} w_{3}\right) p_{1}-w_{3}\left(w_{2}+a w_{3}\right)=0$, 
and the class equation of the congruence reduces to

(9) $\varphi(w) \equiv\left(w_{2}^{2}+a w_{1} w_{3}\right)^{2}+4 w_{2} w_{3} w_{4}\left(w_{2}+a w_{3}\right)=0$.

It is known that every linear complex for which $(p q) \neq 0$ can be reduced* by projective transformation to the canonical form $(p q)+1=0$. Hence we may take as a general case the congruence given by the general $f_{n}(p, q)$ and the special $f_{1}$ of $(7)$.

It follows that $F\left(p_{1}\right)$ in general has coefficients which are homogeneous expressions of order $2 n$ in $w_{i}(i=1,2,3,4)$. Hence since the discriminant of $F\left(p_{1}\right)$ is of degree $2(n-1)$, the class of the general congruence $C(n ; 1)$ is $m=4 n(n-1)$.

In the special case above the class has been reduced by dividing out powers of $w_{i}$.

\section{§4. Generalization to Congruence $C(n ; m)$.}

Instead of the non-homogeneous system of Plücker $\left(p_{i}, q_{i}, r\right)$ we may use the homogeneous coordinates of Grassmann, viz.,

where

$$
\left(q_{12}, q_{13}, q_{14}, q_{23}, q_{42}, q_{34}\right) \text {, }
$$

$$
q_{12} q_{34}+q_{13} q_{42}+q_{14} q_{23}=0 .
$$

Then a congruence $C(n ; m)$ is given by the two homogeneous equations

$$
f_{n}\left(q_{i k}\right)=0, \quad g_{m}\left(q_{i k}\right)=0 .
$$

To obtain the lines in which an arbitrary $w_{x}=0$ intersects $C(n ; m)$ let $u_{x}$ be an arbitrary plane cutting $w_{x}$ in an element of the complex $f_{n}$. Then

$$
q_{i k}=u_{i} w_{k}-u_{k} w_{i},
$$

and the planar equation of the lines of the complex which are contained in $w_{x}=0$ is

$$
f_{n}\left(u_{i} w_{k}-u_{k} w_{i}\right)=0,
$$

where $u_{j}$ are the variables. Likewise $w_{x}=0$ intersects the other complex in the lines

$$
g_{m}\left(u_{i} w_{k}-u_{k} w_{i}\right)=0 .
$$

The lines (11) envelope a curve of class $n \dagger$ and (12) a curve of

* Lie, Die Berührungstransformationen, p. 223.

† Lie, ibid., p. 286. 
class $m$. Hence $w_{x}=0$ cuts $C(n ; m)$ in an nm-line, by Bezout's theorem.*

If we project the curves (11), (12) upon the $x_{3}$ plane, we may obtain the equation of the projected $m n$-line by interchanging point and line coordinates in Clebsch's proof of Bezout's theorem (see Vorlesungen über Geometrie, page 282). Every full invariant of this $m n$-line gives by our translation principle an equation of condition among the coordinates $w_{i}$.

An alternative method of procedure is to use equations (1), (3), replacing $f_{1}$ by $g_{m}$ in (1). Rational elimination processes give a form in each variable $p_{1}, q_{1}, p_{2}, q_{2}$ with coefficients rational in $w_{i}$. Of these forms that in $p_{2}$ is the transformed of the one in $p_{1}$, say of $F_{1}\left(p_{1}\right)$, by a homographic transformation, and that in $q_{2}$ is likewise the transformed of the one in $q_{1}$, viz. $\varphi_{1}\left(q_{1}\right)$. But $\varphi_{1}$ is not transformable into $F_{1}$. As an invariant of the $m n$-line of intersection we may then select a simultaneous invariant of the binary forms $F_{1}\left(p_{1}\right), \varphi_{1}\left(q_{1}\right)$, and by translation this invariant goes into an equation of condition in the variables $w_{i}$, representing a contravariant surface.

University of Pennsyluania, Philladelphia, Pa.

\section{SOME MATHEMATICAL BOOKLET SERIES.}

Matematica dilettevole e curiosa. Di Italo Ghensi. Con 693 figure originali dell'Autore. Milano, Ulrico Hoepli, 1913. viii+730 pp. Price L. 9.50.

Wo steckt der Fehler? Trugschlüsse und Schülerfehler. Gesammelt von Dr. W. Lietzmann und V. Trier. Mathematische Bibliothek, Nr. 10. Leipzig and Berlin, B. G. Teubner, 1913. 57 pp. Price M. 0.80 .

ENGLish and French mathematical literature is entirely lacking in such admirable booklets dealing with elementary topics, as those which have wide circulation in Germany and Italy. $\dagger$ I refer to the Mathematische Bibliothek of the

* Bezout, Theorié générale des Equations algébriques (1779).

$\dagger$ It may be suggested that the volumes on Elimination by Laurent and on Geometrography by Lemoine, of the excellent "Scientia" series (Gauthier-Villars, Paris) are elementary, but these are only two of a dozen volumes by Appell, Gibbs, Hadamard, Poincaré, etc., which certainly may not be classed in this way. And even these two brochures are more 